University of Wollongong

Research Online

Faculty of Engineering and Information

Faculty of Engineering and Information

Sciences - Papers: Part B

Sciences

2020

Tunable strong photo-mixing in Weyl semimetals

Jack Zuber

University of Wollongong, jwz966@uowmail.edu.au

T Zhao

S Gong

$\mathrm{MHu}$

R Zhong

See next page for additional authors

Follow this and additional works at: https://ro.uow.edu.au/eispapers1

Part of the Engineering Commons, and the Science and Technology Studies Commons

Research Online is the open access institutional repository for the University of Wollongong. For further information contact the UOW Library: research-pubs@uow.edu.au 


\title{
Tunable strong photo-mixing in Weyl semimetals
}

\author{
Abstract \\ We study both the temperature and band-gap dependence of the first-and third-order intraband \\ conductivities of a Weyl semimetal (WSM) as well as the resultant critical field. By a semiclassical model \\ we further explore how different chemical potentials, cone separations, field geometries, and finite \\ relaxation times affect photoresponse. We determine that the band-gap variation is characterized by the \\ Weyl cone separation and observe significant thermal enhancement of the nonlinear response. Universal \\ behaviors of both the linear and nonlinear response have also been observed under certain conditions. \\ Coupled with experimentally accessible critical field values of 104-105 V m-1, our results give strong \\ impetus for the use of WSMs as tunable photo-mixers. \\ Disciplines \\ Engineering | Science and Technology Studies

\section{Publication Details} \\ Zuber, J. W., Zhao, T., Gong, S., Hu, M., Zhong, R. B., Zhang, C. \& Liu, S. G. (2020). Tunable strong photo- \\ mixing in Weyl semimetals. Physical Review B, 101 (8), 085307-1-085307-11.

\section{Authors} \\ Jack Zuber, T Zhao, S Gong, M Hu, R Zhong, C Zhang, and S Liu
}




\title{
Tunable strong photo-mixing in Weyl semimetals
}

\author{
J. W. Zuber, ${ }^{1}$ T. Zhao, ${ }^{2,3}$ S. Gong, ${ }^{2,3}$ M. Hu, ${ }^{2,3}$ R. B. Zhong, ${ }^{2,3}$ C. Zhang $\odot,,^{1,4, *}$ and S. G. Liu $^{2,3}$ \\ ${ }^{1}$ School of Physics, University of Wollongong, Wollongong, New South Wales 2522, Australia \\ ${ }^{2}$ Terahertz Research Center, School of Physical Electronics, \\ University of Electronic Science and Technology of China, Chengdu 610054, China \\ ${ }^{3}$ Key Laboratory of Terahertz Technology, Ministry of Education, Chengdu 610054, China \\ ${ }^{4}$ Institute for Superconducting and Electronic Materials, University of Wollongong, Wollongong, New South Wales 2522, Australia
}

(Received 27 June 2019; accepted 19 February 2020; published 28 February 2020)

\begin{abstract}
We study both the temperature and band-gap dependence of the first- and third-order intraband conductivities of a Weyl semimetal (WSM) as well as the resultant critical field. By a semiclassical model we further explore how different chemical potentials, cone separations, field geometries, and finite relaxation times affect photoresponse. We determine that the band-gap variation is characterized by the Weyl cone separation and observe significant thermal enhancement of the nonlinear response. Universal behaviors of both the linear and nonlinear response have also been observed under certain conditions. Coupled with experimentally accessible critical field values of $10^{4}-10^{5} \mathrm{~V} \mathrm{~m}^{-1}$, our results give strong impetus for the use of WSMs as tunable photo-mixers.
\end{abstract}

DOI: 10.1103/PhysRevB.101.085307

\section{INTRODUCTION}

Weyl semimetals (WSMs) are a class of material that can be regarded as three-dimensional analogs of graphene without time-reversal symmetry. This symmetry is broken by the separation of two three-dimensional Dirac (Weyl) cones in momentum space. The two Weyl cones are distinguished by their chirality. The nomenclature of WSMs stems from the Weyl equation used to describe the electronic charge carriers. The electrons near the Weyl points behave like massless charge carriers known as Weyl fermions. Although the Weyl fermion continues to prove elusive in experimental observation [1-4], the general thermal transport [5,6] and charge transport [7-13] properties of WSMs have been investigated in depth both theoretically and experimentally [14-16].

The interaction of two Weyl cones of opposite chirality provides many additional interesting topological properties. Phenomena such as the chiral magnetic effect (due to interplay between bulk Weyl cones and surface Fermi arcs) [17], Andreev-Bragg reflection, the formation of Majorana modes via connectivity of the Fermi arcs, and the well-known chiral anomaly $[18,19]$ (which gives rise to negative magneto-optical resistance $[20,21]$ and ultrahigh carrier mobility [22]) have all been studied in depth.

Due to the similarities in band structure between Dirac and Weyl systems, Weyl systems share some common transport properties with Dirac materials such as suppressed backscattering [23,24], creation of midgap states [25], universal minimum conductivity, Anderson localization [26], and the quantum Hall effect $[27,28]$. However, due to the large bulk contributions to the electron transport this three-dimensional system is more difficult to model.

In the present study we are interested in modeling the nonlinear optical conductivity of a WSM and a gapped WSM

\footnotetext{
*czhang@uow.edu.au
}

where a finite band gap is present. The nonlinear optical conductivity was investigated previously for this material using a Feynman diagrammatic approach where higherorder harmonics were observed [29] and experimentally for transition-metal monopnictide WSMs such as TaAs [30-32]. However, the dependence of the nonlinear response on important parameters such as Weyl cone separation and band gap has not yet been identified. We adopt a robust semiclassical method and scale it up to encompass the more complicated structure of a WSM. By Taylor expanding the velocity operator one is able to calculate the nonlinear conductivities and identify their dependence on the aforementioned intrinsic parameters.

The nonlinear optical properties are characterized by the electric field strength required so that the first- and third-order conductivities are equal in magnitude; this is known as the critical field. If the critical field is experimentally reachable for terahertz frequencies there are a number of potential uses for this material such as photo-mixing and tunable terahertz signal generating and receiving.

\section{MODELS}

The effective (minimal coupling) Hamiltonian for a lowenergy carrier near the Weyl point is described by the Hamiltonian $[8,10]$

$$
H=\left(\begin{array}{cc}
v_{F} \sigma \cdot(\mathbf{p}+\mathbf{b}) & \Delta \mathbb{I}_{2} \\
\Delta \mathbb{I}_{2} & v_{F} \sigma \cdot(\mathbf{b}-\mathbf{p})
\end{array}\right),
$$

where $v_{F} \approx 10^{6} \mathrm{~m} \mathrm{~s}^{-1}$ is the Fermi velocity [33], $\sigma$ are the Pauli matrices, $\mathbf{p}$ is the momentum vector, $\mathbf{b}$ is an intrinsic parameter separating the two opposite-chirality Weyl points in momentum space, and $\Delta$ is a parameter which can gap the dispersion and produce chiral mixing. The parameter $\Delta$ may be intrinsic or introduced manually by doping, for example; conversely, $\mathbf{b}$ is unique to each WSM compound. If $\mathbf{b}=0$ 


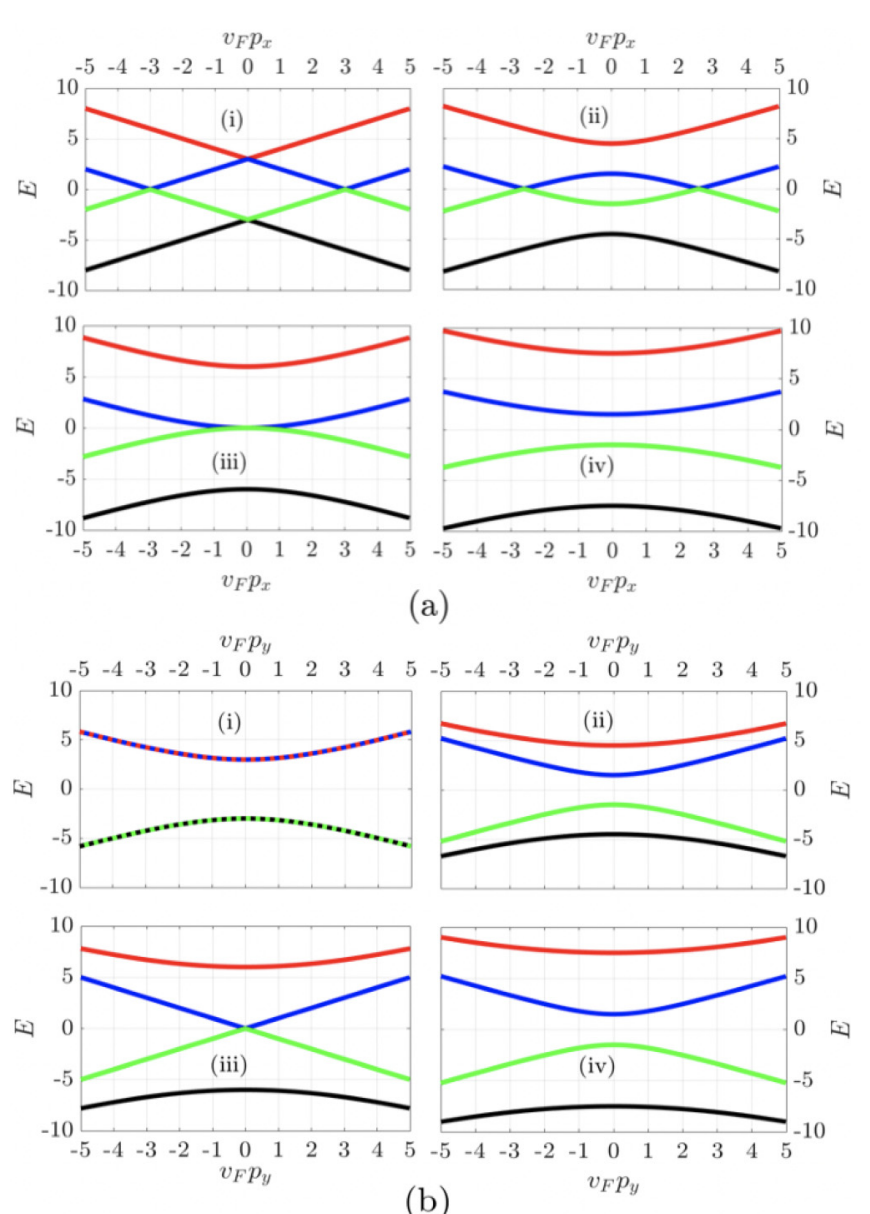

(b)

FIG. 1. WSM dispersion (relative units) with $s=1, r=1$ (red), $s=1, r=-1$ (blue), $s=-1, r=-1$ (green), $s=-1, r=1$ (black), and $v_{F} \mathbf{b}=v_{F} b_{x}=3$. The plots are along (a) $p_{y}=p_{z}=0$ and (b) $p_{x}=p_{z}=0$. The subpanels have the following parameter ensembles: (i) $\Delta=0$, (ii) $\Delta=1.5$, (iii) $\Delta=3$, and (iv) $\Delta=5$.

the Hamiltonian is $C P T$ invariant [8,9]; however, $\mathbf{b} \neq 0$ breaks time-reversal symmetry and the isotropy of the dispersion (see Fig. 1).

The Hamiltonian in Eq. (1) is readily diagonalized to produce the following four-band dispersion:

$$
\begin{aligned}
E_{s, r}= & s v_{F} \\
& \times \sqrt{\left(\frac{\Delta}{v_{F}}\right)^{2}+b^{2}+p^{2}+2 r \sqrt{(\mathbf{b} \cdot \mathbf{p})^{2}+\left(\frac{\Delta b}{v_{F}}\right)^{2}}},
\end{aligned}
$$

where $p=|\mathbf{p}|, b=|\mathbf{b}|$, and $s, r= \pm 1$. The parameter $s$ is an analog to charge conjugation symmetry in quantum electrodynamics [34] and is used to distinguish between the conduction bands $(s=1)$ and valence bands $(s=-1)$.

The parameter $r$ has a more intricate effect on the dispersion. The bands described by $r=1$ are analogous to a gapped Dirac-like structure with extrema located at $\mathbf{p}=0$ with value $E_{s, r=1}^{\mathrm{ex}}=s\left(b+\frac{\Delta}{v_{F}}\right)$. The $r=-1$ bands encode a much more interesting topology. When $\left(\frac{\Delta}{v_{F}}\right)^{2}>b^{2}$ the $r=-1$ valence and conduction bands will be gapped [fully gapped system,

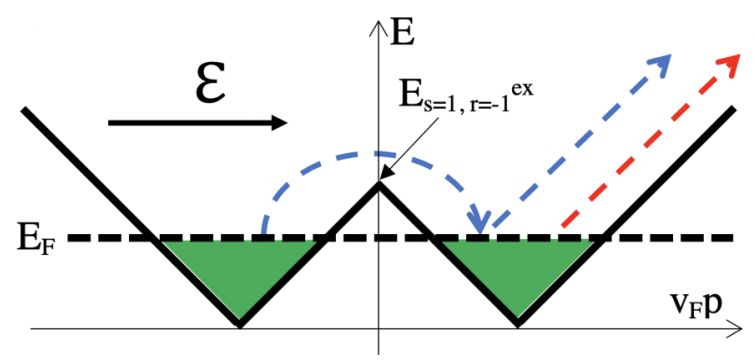

FIG. 2. Two types of contributions to the intraband response: blue arrow, intercone contribution; red, conventional intraband response. The green areas represent the regions of charge carriers we consider with $E_{F}$ the Fermi level and $\mathcal{E}$ the applied field.

Fig. 1(aiv)]; however, when $\left(\frac{\Delta}{v_{F}}\right)^{2}<b^{2}$ the dispersion exhibits two distinct Weyl cones [partially gapped system, Fig. 1(aii)] with Weyl points separated in momentum space by the vector $2 \sqrt{1-\left(\frac{\Delta}{v_{F} b}\right)^{2}} \hat{b}$. In either case there are extremal points at

$$
\left(\mathbf{p}, E_{s, r=-1}^{\mathrm{ex}}\right)=\left(\mathbf{0}, s v_{F}\left|\frac{\Delta}{v_{F}}-b\right|\right) .
$$

Without loss of generality, we choose $\mathbf{b}$ to be oriented along one axis, say, $\mathbf{b}=\left(b_{x}, 0,0\right)$. Dispersions along the momentum direction parallel to $\mathbf{b}$ [Fig. 1(a)] and perpendicular to $\mathbf{b}$ [Fig. 1(b)] are shown in Fig. 1.

It is readily observed that the $r=1$ (black and red) bands increase linearly in separation with $\Delta$ according to $2\left(v_{F} b+\right.$ $\Delta)$. These bands also become more diffuse or flat with an increase in $\Delta$. This flattening is caused by a renormalization of the Fermi velocity and yields a higher carrier mobility in this direction.

Similarly, flattening also occurs for the $r=-1$ bands. Furthermore, as $\Delta$ increases, the two Weyl points converge uniformly until they become doubly degenerate when $v_{F} b=$ $\Delta$ as in Fig. 1(aiii). If a field were to be directed along $p_{x}$, intercone transitions (see Fig. 2) would become more dominant as the cones moved closer together, mixing chiral states, until the Weyl points collapse. The decay of the local maxima $E_{s=1, r=-1}^{\mathrm{ex}}$ with $\Delta$ also aids said intercone transitions or chiral transport, allowing charge carriers to flow more freely between Weyl cones when $E_{s=1, r=-1}^{\mathrm{ex}}$ dips below the Fermi level.

Along a direction perpendicular to $\mathbf{b}$ we find that the $r=$ 1 bands behave in an identical manner when increasing $\Delta$. However, the $r=-1$ bands appear as a doubly degenerate gapped Dirac-like structure for $\Delta=0$ as they are separated in a perpendicular direction and move together to form a single Weyl point when $v_{F} b=\Delta$, then move back apart as $\Delta \rightarrow \infty$. Intercone transitions are still aided by the convergence of the Weyl cones.

We consider the material subject to a monochromatic and homogeneous time-dependent electric field in the $x$ direction given by

$$
\mathcal{E}(t)=\mathcal{E} e^{-i \omega t} \hat{x} .
$$

This external field is coupled to the quasiparticles via the Pierel substitution $\mathbf{p} \rightarrow \mathbf{p}+e \mathbf{A}(t)$, where $\mathcal{E}=-\partial \mathbf{A}(t) / \partial t$ and $e$ is the electron charge. The velocity operator is given 
as [34]

$$
\langle\mathbf{v}(\mathbf{p}+e \mathbf{A})\rangle=\left\langle\nabla_{\mathbf{p}} H(\mathbf{p}+e \mathbf{A})\right\rangle=\nabla_{\mathbf{p}} E(\mathbf{p}+e \mathbf{A}) .
$$

In the velocity expression we omitted the the anomalous term related to the Berry curvature, $\dot{k} \times \Omega$. Since the Chern numbers of the two Weyl points are \pm 1 , the sum of the anomalous terms has no contribution to the total current. For an applied field along the $x$ direction, we are only concerned with the $x$ component of the velocity operator. A basic Taylor expansion allows us to expand the velocity operator in orders of $\mathbf{A}$. We obtain

$$
\begin{gathered}
v_{x}^{(0)}=s v_{F}\left[\frac{A}{B^{\frac{1}{2}}}\right] \\
\frac{v_{x}^{(1)}}{e A_{x}}=s v_{F}\left[\frac{B C-A^{2}}{B^{\frac{3}{2}}}\right] \\
\frac{v_{x}^{(2)}}{\left(e A_{x}\right)^{2}}=-\frac{3 s v_{F}}{2}\left[\frac{(\mathbf{b} \cdot \mathbf{p}) B^{2} D+A\left(B C-A^{2}\right)}{B^{\frac{5}{2}}}\right], \\
\frac{v_{x}^{(3)}}{\left(e A_{x}\right)^{3}}=\frac{s v_{F}}{2}\left[\frac{4(\mathbf{b} \cdot \mathbf{p}) A B^{2} D+5(\mathbf{b} \cdot \mathbf{p})^{2} B^{3} F-3 b_{x} B^{3} D}{B^{\frac{7}{2}}}\right] \\
\left.-\frac{C^{2} B^{2}-6 A^{2} B C+5 A^{4}}{B^{\frac{7}{2}}}\right]
\end{gathered}
$$

where

$$
\begin{gathered}
A=p_{x}+r \frac{b_{x}(\mathbf{b} \cdot \mathbf{p})}{\sqrt{(\mathbf{b} \cdot \mathbf{p})^{2}+\left(\frac{\Delta}{v_{F}} b\right)^{2}}}, \\
B=\left(\frac{\Delta}{v_{F}}\right)^{2}+b^{2}+p^{2}+2 r \sqrt{(\mathbf{b} \cdot \mathbf{p})^{2}+\left(\frac{\Delta}{v_{F}} b\right)^{2}}, \\
C=1+r \frac{\left(b_{x} b \frac{\Delta}{v_{F}}\right)^{2}}{\left((\mathbf{b} \cdot \mathbf{p})^{2}+\left(\frac{\Delta}{v_{F}} b\right)^{2}\right)^{\frac{3}{2}}}, \\
D=r \frac{b_{x}^{3}\left(\frac{\Delta}{v_{F}} b\right)^{2}}{\left((\mathbf{b} \cdot \mathbf{p})^{2}+\left(\frac{\Delta}{v_{F}} b\right)^{2}\right)^{\frac{5}{2}}},
\end{gathered}
$$

and

$$
F=r \frac{b_{x}^{4}\left(\frac{\Delta}{v_{F}} b\right)^{2}}{\left((\mathbf{b} \cdot \mathbf{p})^{2}+\left(\frac{\Delta}{v_{F}} b\right)^{2}\right)^{\frac{7}{2}}} .
$$

The $n$ th-order current can then be obtained from

$$
J_{x}^{(n)}=\frac{e}{(2 \pi \hbar)^{3}} \sum_{s, r} \int v_{x}^{(n)} N_{F}\left(E_{s, r}, E_{F}, T\right) d^{3} \mathbf{p} .
$$

The limits of integration for $r=-1$ are chosen so as to avoid the singular Weyl points where the assumption that our model is based on, $p \gg e|\mathbf{A}|$, fails. We do so by removing a cube from the integration domain that is centered at the Weyl point with the length from the Weyl point to the corner of the cube (half diagonal), $\frac{E_{p h}}{v_{F}}=\frac{\hbar \omega}{v_{F}}$.

$N_{F}=\left(1+\exp \left[\left(E_{S, r}-E_{F}(\mathbf{b}, \Delta)\right) T^{-1}\right]\right)^{-1}$ is the FermiDirac distribution function where the Fermi level $E_{F}$ is recalculated when changing $(\mathbf{b}, \Delta)=\left(\mathbf{b}_{i}, \Delta_{i}\right) \rightarrow\left(\mathbf{b}_{n}, \Delta_{n}\right)$ by

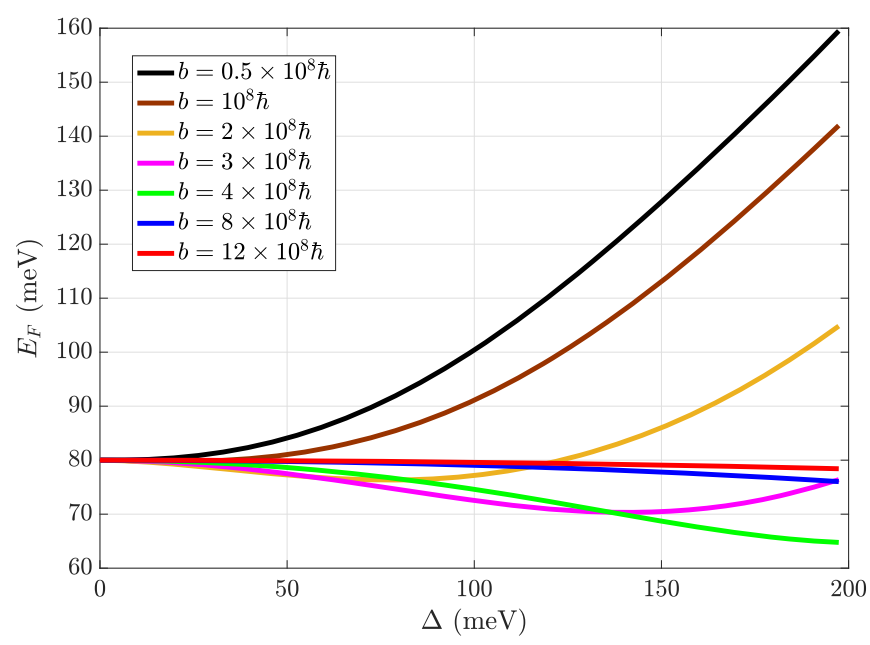

FIG. 3. Dependence of Fermi level on different initial cone separations with $\mu_{0}=80 \mathrm{meV}$.

numerically solving the equation

$$
\begin{aligned}
\int_{0}^{\infty} d E \int_{\mathbb{R}^{3}} d^{3} \mathbf{p} \delta\left(E_{s, r}\left(\mathbf{p}, \mathbf{b}_{n}, \Delta_{n}\right)-E\right) N_{F}\left(E, E_{F}, T\right) \\
\quad=\int_{0}^{\infty} d E \int_{\mathbb{R}^{3}} d^{3} \mathbf{p} \delta\left(E_{s, r}\left(\mathbf{p}, \mathbf{b}_{i}, \Delta_{i}\right)-E\right) N_{F}\left(E, \mu_{0}, T\right),
\end{aligned}
$$

with $\mu_{0}$ taken as the (intrinsic) chemical potential of the material. This process is followed to ensure that the carrier density, an intrinsic property of the material, stays constant. The characteristics of $E_{F}$ are shown explicitly in Sec. III A.

Finally, since we are interested in the electronic intraband conductivity and for typical parameters $[12,33,35]$ $\mu_{0} \ll E_{s=1, r=1}$ we only consider the contribution from the $s=1, r=-1$ band. In neglecting the contribution from other bands, the special case of $\mathbf{b}=0$ will mimic a threedimensional (3D) Dirac material which then becomes gapped for $\Delta>0$. For consistency with the Hamiltonian presented in Eq. (1) we still refer to this structure as a gapped WSM.

We note that gap opening leads to the internode coupling. As a result, the topologically protected transport is suppressed. Our purpose here is not to study the topologically protected transport, but the nonlinear photo-mixing due to the gap opening and Weyl points separation. Although a gap opening suppresses topological order, it plays an important role to enhance the strong mixing effect. This can be of technological importance in potential application of WSM in photonics.

\section{RESULTS}

The $\mathbf{b}$ values used are based on $k_{\mathbf{b}}=0.08 \AA^{-1}$ for TaAs [13] with the $\Delta$ range allowing us to view the features of both the partially and fully gapped band structures. We chose relatively high $\mu$ values (of the order $100 \mathrm{meV}$ ) to ensure the assumption $|\mathbf{p}| \gg e|\mathbf{A}|$ is valid.

\section{A. Fermi level}

Figure 3 reveals a three-stage behavior of the Fermi level. First, for large $b$, when the two Weyl points are far apart 


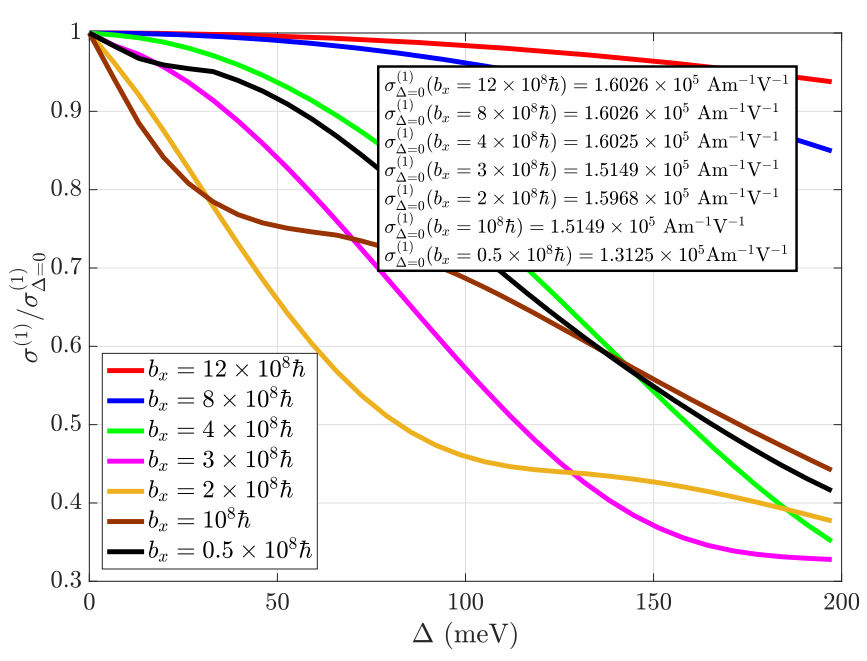

FIG. 4. First-order conductivity with $\mu_{0}=80 \mathrm{meV}$ and $T=$ $300 \mathrm{~K}$ with $\Delta=0 \mathrm{meV}$ values obtained for $\omega=1 \mathrm{THz}$.

and $E_{s=1, r=-1}^{\mathrm{ex}}>E_{F}$, the Fermi level decreases with $\Delta$. This is because, as the Weyl points move together and $E_{s=1, r=-1}^{\mathrm{ex}}$ decreases (at a rate faster than $E_{F}$ ), the carrier density will increase. Such behavior persists until $E_{s=1, r=-1}^{\mathrm{ex}}=E_{F}$ and the Fermi level reaches a minimum. This minimum is evident over the $\Delta$ range displayed for $b=2 \times 10^{8} \hbar$ (at approximately $\Delta=65 \mathrm{meV}$ ) and $b=10^{8} \hbar$ (at approximately $\Delta=$ $130 \mathrm{meV}$ ). Once $E_{s=1, r=-1}^{\mathrm{ex}}<E_{F}$, the Fermi level begins increasing indefinitely into the region where $\Delta$ becomes large enough to completely gap the dispersion as expected. Furthermore, we note importantly that the Fermi level variations are independent of the direction of $\mathbf{b}$. This is a remnant of the freedom of choice of a coordinate system in the absence of an external, directional-dependent, field.

Finally, we remark that changing the chemical potential has no affect on the dynamical behavior of the Fermi level. The Fermi level is merely shifted downwards or upwards in accordance with said change in chemical potential. This is expected as shifting the chemical potential should result in a constant shift in the carrier density which is then maintained as $\Delta$ changes.

\section{B. Linear optical response}

We begin by considering the band-gap parameter $\Delta$ as the independent variable. In Fig. 4 all curves decrease as $\Delta$ increases. This is expected as gapped systems should become worse conductors. Furthermore, we observe that smaller $\mathbf{b}$ values produce smaller $\Delta=0$ conductivities, reaching a plateau at $1.6026 \times 10^{5} \mathrm{~A} \mathrm{~m}^{-1} \mathrm{~V}^{-1}$. Such a magnitude correlates well with similar materials (graphite, $(2-3) \times 10^{5} \mathrm{~A} \mathrm{~m}^{-1} \mathrm{~V}^{-1}$ ).

The rate of decrease depends heavily on $b$. When the dispersion is partially gapped $\left(v_{F} b>\Delta\right)$, smaller $b$-value curves decrease more rapidly. This is because the Weyl points are easier to collapse; i.e., the spectrum becomes gapped for a smaller $\Delta$. Moreover, we find that there is a point of inflection that occurs when $v_{F} b=\Delta$ on each curve (this point is outside the $\Delta$ range for the three largest $b$ values). This topologically significant point marks the $\Delta$ value at which the Weyl points collapse, whereby the intercone contribution reaches and stays

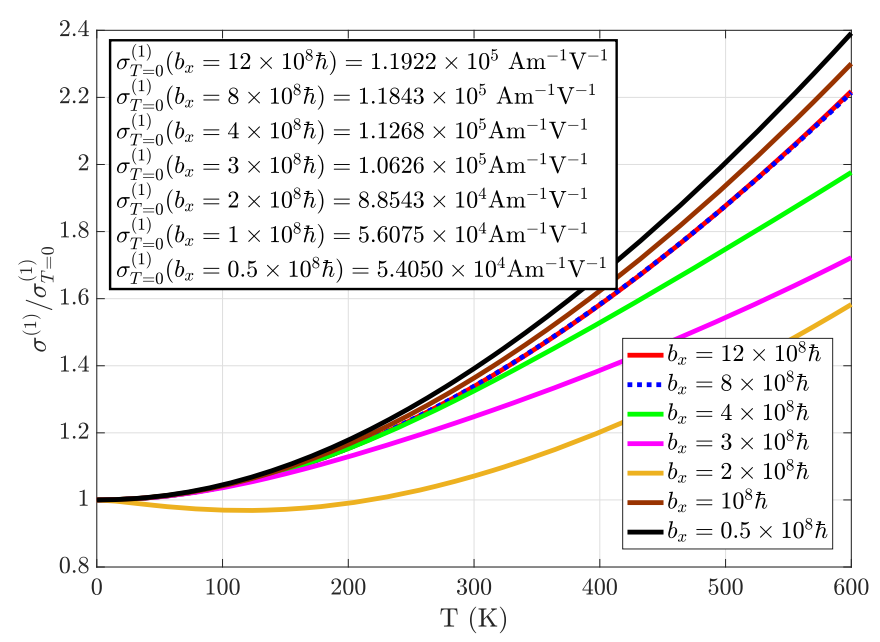

FIG. 5. First-order conductivity with $\mu_{0}=80 \mathrm{meV}$ and $\Delta=$ $53 \mathrm{meV}$ with $T=0 \mathrm{~K}$ values obtained for $\omega=1 \mathrm{THz}$.

at a maximum. For larger values of $\Delta$, smaller $b$ curves again decrease more rapidly until reaching a plateau at $\sigma^{(1)}=0$.

Our results reveal an interesting universal behavior. The chemical potential and temperature do not affect the $\Delta$ dependence of the first-order conductivity. For the fixed value $b=b_{x}=8 \times 10^{8} \hbar$ all curves follow the universal trend

$$
\frac{\sigma^{(1)}(\Delta)}{\sigma_{\Delta=0}^{(1)}} \approx 1-\alpha \Delta^{2},
$$

where $\alpha \approx 3.7 \times 10^{-6}$ is independent of $\mu_{0}, T$, and $\omega$. Referring to Fig. 4, we note that the quadratic shape of the scaled first-order conductivity is not universal for all $b$ values. The independence from $\mu_{0}$ is expected since we have taken into account how $E_{F}$ changes with $\Delta$ (and hence b). However, results for $\sigma_{\Delta=0}^{(1)}$ still show that smaller chemical potentials provide a smaller-magnitude conductivity as expected physically due to the lack of available charge carriers at low chemical potentials.

On the other hand, the independence on temperature in Eq. (8) is accurate to within $2 \%$ for $T \in[0,600] \mathrm{K}$ and $\Delta \in$ $[0,200]$ meV-higher temperatures decrease slightly less rapidly with $\Delta$. The $\Delta=0$ values show that higher temperatures produce higher first-order conductivities in this regime. One should expect a higher first-order conductivity for higher temperatures purely from a carrier distributional sense.

We remark that due to the universal $\Delta$ dependence for chemical potential and temperature shown in Eq. (8) that $\mathbf{b}$ plays the most influential role in the $\Delta$-dependent first-order conductivity. The temperature dependence is shown in Fig. 5.

The Weyl cone separation parameter plays an interesting role in the temperature dependence of the first-order conductivity. First, the $T=0 \mathrm{~K}$ values are appreciably smaller for smaller $b$ values due to the fixed $\Delta$. Second, in general, the first-order conductivities increase approximately exponentially with temperature. Such an increase has been seen using similar models for graphene [34].

The rate of increase, however, is strikingly dependent on $b$. It begins to decrease as $E_{F}$ decreases until it reaches a minimum for $b_{x}=2 \times 10^{8} \hbar$ whereby $E_{F} \approx E_{s=1, r=-1}^{\mathrm{ex}}$ and 


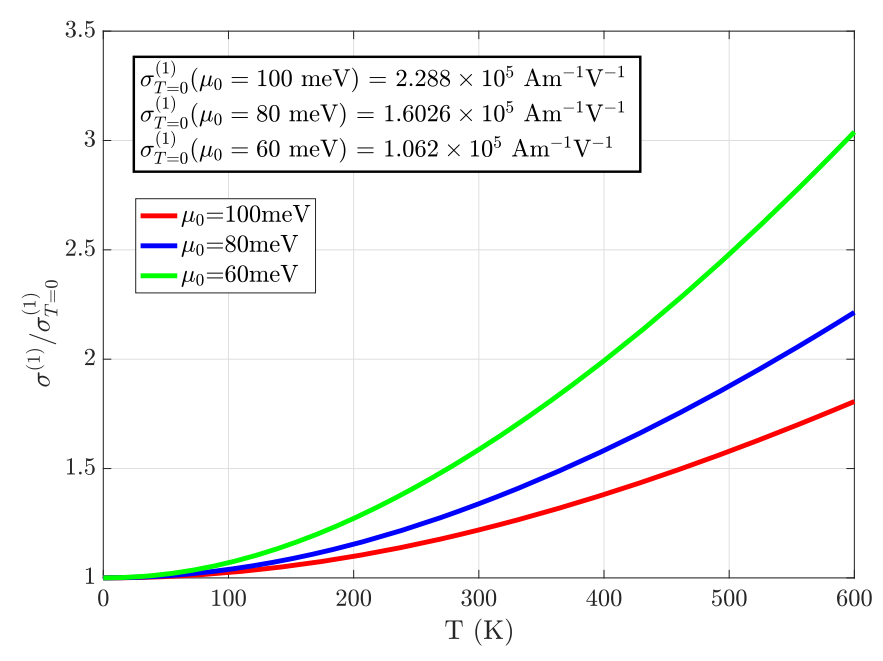

FIG. 6. First-order conductivity with $b=b_{x}=8 \times 10^{8} \hbar$ and $\Delta=53 \mathrm{meV}$ with $T=0 \mathrm{~K}$ values obtained for $\omega=1 \mathrm{THz}$.

there is in fact a region of lower conductivity between 0 and $200 \mathrm{~K}$. Thereafter when $b$ forces $E_{F}>E_{s=1, r=-1}$ the rate of increase with temperature becomes greater.

Figure 6 shows that $\mu_{0}$ plays a significant role in altering the first-order conductivity magnitude as a function of temperature. Smaller $\mu_{0}$ values yield a faster rate of increase of the conductivity with temperature but a smaller conductivity magnitude as evidenced by the $T=0$ values. Again, the magnitude is dictated by the availability of charge carriers while the rate of increase is larger for deeper (lower-energy) carriers.

Finally, it can be shown that the temperature dependence of the first-order conductivity is not dependent on $\Delta$. For $b=b_{x}=8 \times 10^{8} \hbar$, all curves take the universal value

$$
\frac{\sigma^{(1)}(T)}{\sigma_{T=0}^{(1)}}=1+\alpha T^{2},
$$

where the parameter $\alpha$ is identical to that introduced in Eq. (8).

Consistent with previous results $[8,9,29]$ we find that the second-order current response vanishes regardless of the magnitudes of $\Delta$ and $\mathbf{b}$. This occurrence can easily be identified mathematically through the spherical symmetry of the second-order velocity operator derived in Eq. (5).

\section{Third-order conductivity (three-photon mixing)}

It has been shown that in two-dimensional materials like graphene there is a strong third-order response in the absence of scattering and gap opening. A three-dimensional Dirac system behaves very differently; the third-order conductivity vanishes in the special case of $v_{F} b=\Delta=0$. This is a direct consequence of the spherically symmetric third-order velocity operator

$$
\begin{aligned}
& v_{x}^{(3)}\left(v_{F} \mathbf{b}=\Delta=0\right) \\
& \quad=-\frac{s r v_{F}\left(e A_{x}\right)^{3}\left(p_{y}^{2}+p_{z}^{2}\right)\left(p_{y}^{2}+p_{z}^{2}-4 p_{x}^{2}\right)}{2 p^{7}} .
\end{aligned}
$$

This result highlights the role dimensionality plays in the intraband conductivity of a graphene-type dispersion $E=$ $v_{F}|\mathbf{p}|$, whereby in two dimensions (conventional graphene)

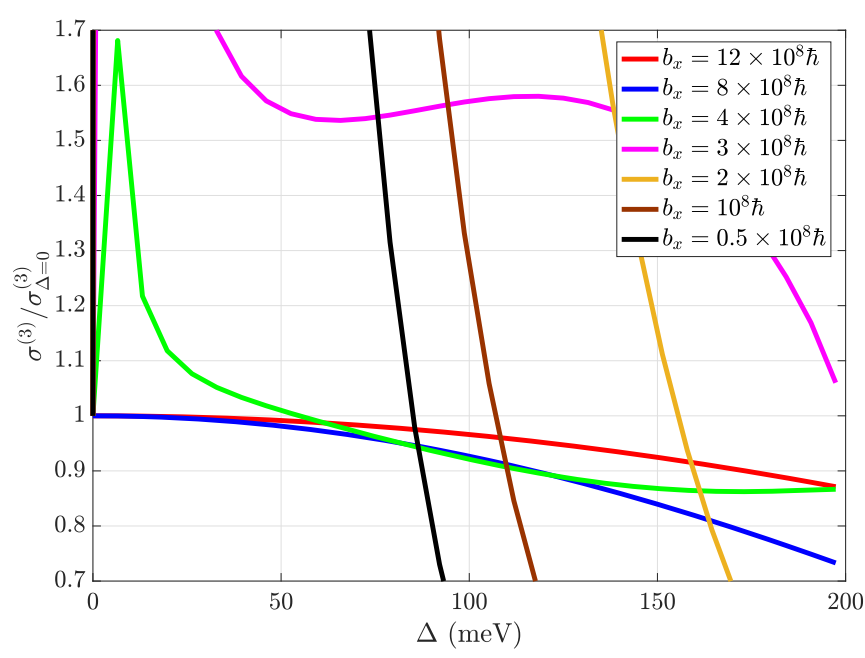

FIG. 7. Third-order conductivity with $\mu_{0}=80 \mathrm{meV}$ and $T=300 \mathrm{~K}$ with $\Delta=0 \mathrm{meV}$ values obtained for $\omega=1 \mathrm{THz}$. The $T=0 \mathrm{~K}$ values are as follows: $\sigma_{\Delta=0}^{(3)}\left(b_{x}=12 \times 10^{8} \hbar\right)=$ $9.2728 \sigma_{0}, \quad \sigma_{\Delta=0}^{(3)}\left(b_{x}=8 \times 10^{8} \hbar\right)=9.2728 \sigma_{0}, \quad \sigma_{\Delta=0}^{(3)}\left(b_{x}=\right.$ $\left.4 \times 10^{8} \hbar\right)=9.2728 \sigma_{0}, \quad \sigma_{\Delta=0}^{(3)}\left(b_{x}=3 \times 10^{8} \hbar\right)=9.5565 \sigma_{0}$, $\sigma_{\Delta=0}^{(3)}\left(b_{x}=2 \times 10^{8} \hbar\right)=9.2346 \sigma_{0}, \quad \sigma_{\Delta=0}^{(3)}\left(b_{x}=10^{8} \hbar\right)=8.2260 \sigma_{0}$, $\sigma_{\Delta=0}^{(3)}\left(b_{x}=0.5 \times 10^{8} \hbar\right)=4.9175 \sigma_{0}$. Here $\sigma_{0}=10^{-6} \mathrm{~A} \mathrm{~m} \mathrm{~V}^{-3}$.

there is a known large third-order response [34], which then disappears in three dimensions (degenerate WSM).

The $\Delta$-dependent nonlinear conductivity is shown in Fig. 7. The initial $\Delta=0$ conductivities are approximately equal for larger $b$ values but decrease rapidly for smaller $b$. The $b_{x}=12 \times 10^{8} \hbar$ and $b_{x}=8 \times 10^{8} \hbar$ third-order conductivity curves decrease with $\Delta$ at a rate decreasing with $b$.

However, for $b_{x}<8 \times 10^{8} \hbar$, the variation appears more interesting. First, there is a large initial spike in the conductivity, the height of which increases for smaller $b$. This spike is attributed to the drastic change in topology (conic to curved) of the dispersion when $\Delta \neq 0$. There are inflections for each curve when $v_{F} b=\Delta$. The inflections for $b_{x}=12 \times 10^{8} \hbar$ and $b_{x}=8 \times 10^{8} \hbar$ are again outside the $\Delta$ range surveyed and are masked by the rapid decrease for $b_{x}=2 \times 10^{8} \hbar, b_{x}=10^{8} \hbar$, and $b_{x}=0.5 \times 10^{8} \hbar$. This rapid decrease is again attributed to the gapping of the dispersion and forces $\sigma^{(3)} \rightarrow 0$ for larger $\Delta$.

The third-order $\Delta$ dependence is found to be insensitive to chemical potential and temperature. For $b=b_{x}=8 \times 10^{8} \hbar$ all curves follow the universal trend

$$
\frac{\sigma^{(3)}(\Delta)}{\sigma_{T=0}^{(3)}} \approx 1-\beta \Delta^{2},
$$

where $\beta=6.75 \times 10^{-6}$. Since $\beta>\alpha$, from Eqs. (8) and (9) it is evident that a band gap inhibits multiple-photon processes more drastically than single-photon processes. Also as in the case of the first order, the magnitudes of $\sigma^{(3)}$ are larger for higher chemical potentials and temperatures. $\mathbf{b}$ is the dominant parameter when considering the third-order conductivity as a function of $\Delta$. Finally, the magnitudes of the initial values $\left(\approx 10^{-6} \mathrm{~A} \mathrm{~m} \mathrm{~V}^{-3}\right)$ are shown to be physically acceptable by the critical fields obtained in Sec. III D. The temperature 


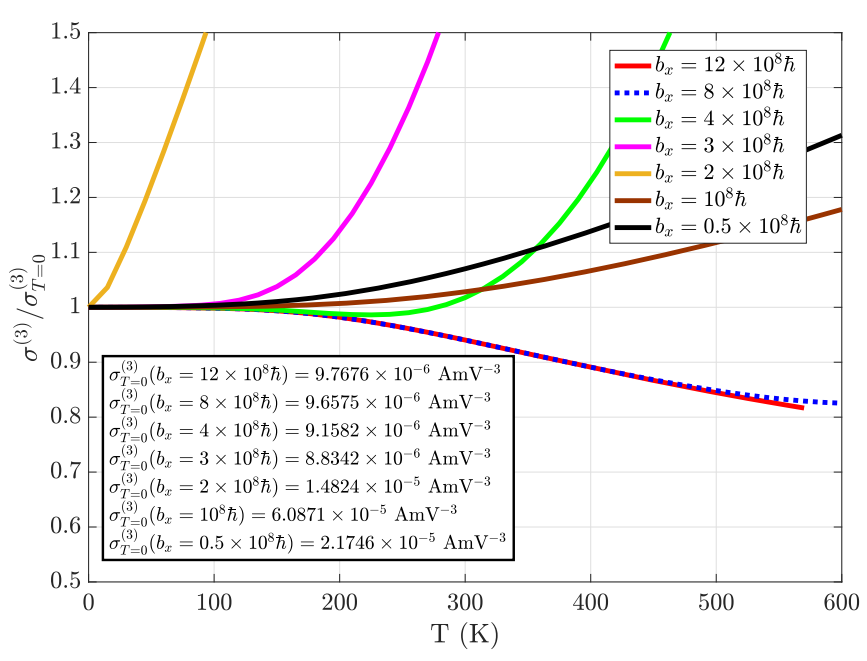

FIG. 8. Third-order conductivity with $\mu_{0}=80 \mathrm{meV}$ and $\Delta=$ $53 \mathrm{meV}$ with $T=0 \mathrm{~K}$ values obtained for $\omega=1 \mathrm{THz}$.

dependence of the nonlinear conductivity is shown in Figs. 8 and 9.

There is a significant increase in the $T=0 \mathrm{~K}$ values for $b=2 \times 10^{8} \hbar$ continuing for $b=10^{8} \hbar$ and dropping off again for $b=0.5 \times 10^{8} \hbar$. The increase occurs when $E_{s=1, r=-1}^{\mathrm{ex}}=$ $E_{F}$ (guaranteeing intercone transitions) and drops off again as $E_{F}$ increases past the extremum (as the intercone contribution plateaus and the conventional intraband contribution lessens).

In terms of the finite-temperature behavior, the two larger b values decrease over this temperature range, with the rate of decrease slowing at higher temperatures. Such a region of decrease occurs as the conventional intraband charge carriers have less chance to couple to three photons at higher temperatures and instead contribute mainly to the monotonically increasing linear response shown in Fig. 5.

Conversely, the $b_{x}=4 \times 10^{8} \hbar$ curve begins by decreasing, reaches a minimum around $T=225 \mathrm{~K}$, then begins to increase rather rapidly - akin to $b_{x}=2 \times 10^{8} \hbar$ and $b_{x}=3 \times$ $10^{8} \hbar$. We hypothesize that the rapid increase is due to thermal

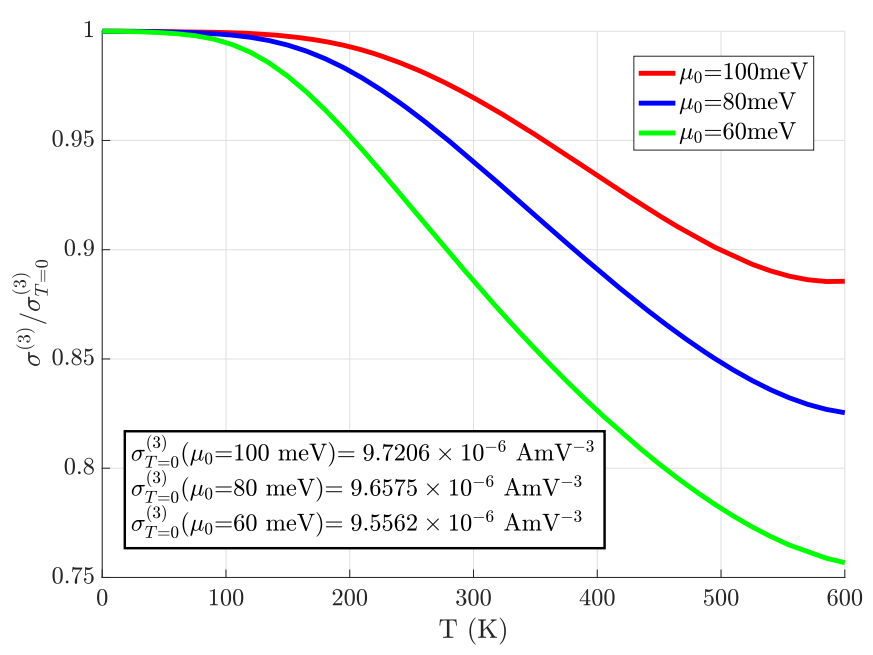

FIG. 9. Third-order conductivity with $b=b_{x}=8 \times 10^{8} \hbar$ and $\Delta=53 \mathrm{meV}$ with $T=0 \mathrm{~K}$ values obtained for $\omega=1 \mathrm{THz}$. activation of intercone transitions, hence why it occurs at smaller temperatures for smaller $b$ values and is guaranteed for ensembles of $(\mathbf{b}, \Delta)$ which place $E_{F}>E_{s=1, r=-1}^{\mathrm{ex}}$. In this way, the monotonic increase of the $b_{x}<4 \times 10^{8} \hbar$ curves is attributed to intercone transitions being thermally activated at $0 \mathrm{~K}$. Furthermore, we expect the $b_{x}=8 \times 10^{8} \hbar$ and $b_{x}=$ $12 \times 10^{8} \hbar$ curves to display this increase at very high temperatures as well.

The curves which decrease over this temperature range are in stark contrast to the first-order conductivity in which there is a thermal enhancement. This is also different from graphene, where the third-order conductivity may reach up to six times its initial $T=0$ value at $300 \mathrm{~K}$ [34].

Figure 9 shows that larger $\mu_{0}$ values give a largermagnitude conductivity and slower rate of decrease. Finally, $\Delta$ plays an almost negligible role in the third-order temperature dependence with the $b=b_{x}=8 \times 10^{8} \hbar$ curve following

$$
\frac{\sigma^{(3)}(T)}{\sigma_{T=0}^{(3)}} \approx \gamma+\gamma e^{-\delta T^{2}},
$$

where $\gamma \approx 0.82$ and $\delta \approx 2.135 \times 10^{-5}$. The magnitudes of $\sigma^{(3)}$ still show a decrease for larger $\Delta$ when the scaling by $\sigma_{T=0}^{(3)}$ is removed as expected.

\section{Critical field}

In nonlinear response the critical field is defined as the field at which the magnitude of the first-order conductivity $\sigma^{(1)}$ is equal to the magnitude of the third-order conductivity $\sigma^{(3)} \mathcal{E}^{2}$ and is hence given by

$$
\mathcal{E}_{c}=\sqrt{\left|\frac{\sigma^{(1)}}{\sigma^{(3)}}\right|} .
$$

From the $\Delta$-dependent data we obtain the following critical field dependence: For the larger two b values, the critical field increases slightly with $\Delta$ as one would expect since the thirdorder conductivity drops off faster than the first order when nearing a gapped dispersion. The smaller $b_{x}=8 \times 10^{8} \hbar$ value increases faster since the dispersion will become gapped for a smaller $\Delta$.

For $b_{x} \leqslant 4 \times 10^{8} \hbar$, however, the critical field has a local minimum as soon as $\Delta$ becomes nonzero, in accordance with the third-order peak. The magnitude of these peaks increases for smaller $b$ but caps out at $\approx 0.1 \times 10^{4} \mathrm{~V} \mathrm{~m}^{-1}$ when the material limit is reached. The critical field for $b_{x}=$ $0.5 \times 10^{8} \hbar$ and $b_{x}=10^{8} \hbar$ then increases for the remainder of the $\Delta$ interval owing to the rapid decrease in third-order conductivity.

Most interestingly, for $b_{x}=2 \times 10^{8} \hbar$ and $b_{x}=4 \times 10^{8} \hbar$ there is a region where the critical field decreases with $\Delta$. This region stems from the point of inflection at $v_{F} b=\Delta$. Once again this point does occur for all $b_{x}>0.8 \times 10^{8} \hbar$ but at higher $\Delta$ for larger $b$.

Physically, this region of decrease is hypothesized to be caused by an accelerant of intercone transitions when $\Delta$ becomes large enough to move the Weyl points close enough so that the $E_{s=1, r=-1}^{\mathrm{ex}}<E_{F}$, but not so large that the dispersion 


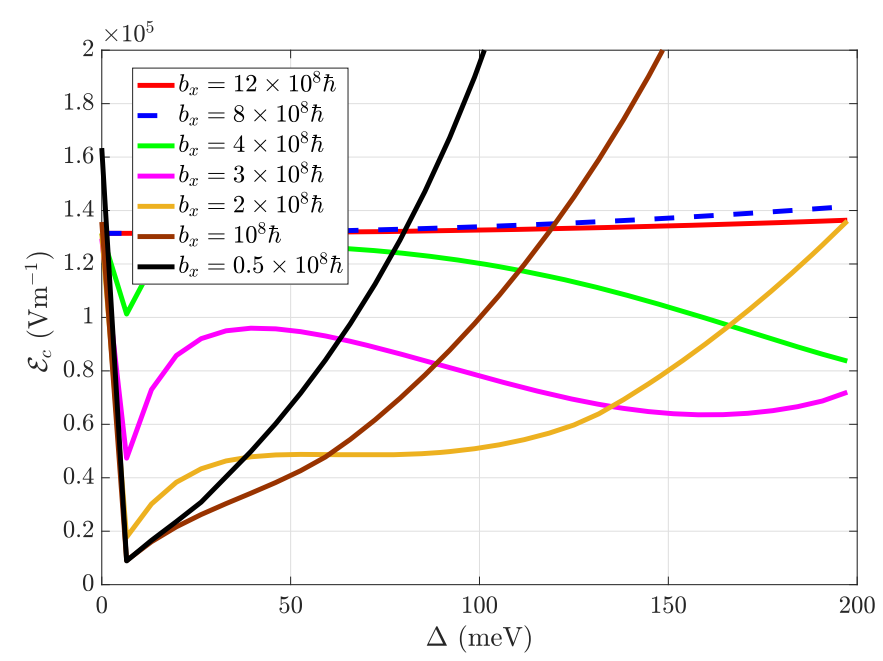

FIG. 10. Critical field with $\omega=1 \mathrm{THz}, \mu_{0}=80 \mathrm{meV}$, and $T=$ $300 \mathrm{~K}$.

is completely gapped. This region would be of most intrigue from an applications standpoint.

Owing to the universal $\Delta$ dependence of the first- and thirdorder conductivities for each value of $\mathbf{b}$ shown in Eqs. (8) and (10), the critical field is simply shifted upwards for larger temperatures and chemical potentials with $\Delta$ dependence given by

$$
\frac{\mathcal{E}_{c}(\Delta)}{\mathcal{E}_{c}(\Delta=0)} \approx \sqrt{\frac{1-\alpha \Delta^{2}}{1-\beta \Delta^{2}}}
$$

which, since $1 \gg \beta>\alpha$, implies that the critical field increases approximately quadratically with $\Delta$ for $b=b_{x}=8 \times$ $10^{8} \hbar$, agreeing with Fig. 10. For reference it was determined that $\mathcal{E}_{c}\left(\Delta=0, T=300 \mathrm{~K}, \mu_{0}=100 \mathrm{meV}\right)=1.54 \times 10^{5}$ $\mathrm{V} \mathrm{m}^{-1}, \mathcal{E}_{c}\left(\Delta=0, T=300 \mathrm{~K}, \mu_{0}=60 \mathrm{meV}\right)=1.11 \times 10^{5}$ $\mathrm{V} \mathrm{m}{ }^{-1}, \mathcal{E}_{c}\left(\Delta=0, T=150 \mathrm{~K}, \mu_{0}=80 \mathrm{meV}\right)=1.16 \times 10^{5}$ $\mathrm{V} \mathrm{m}{ }^{-1}$, and $\mathcal{E}_{c}\left(\Delta=0, T=0 \mathrm{~K}, \mu_{0}=80 \mathrm{meV}\right)=0.68 \times$ $10^{5} \mathrm{~V} \mathrm{~m}^{-1}$.

From Fig. 11 we obtain the following critical fields: For all $b$ values bar $b_{x}>10^{8} \hbar$, the critical field increases initially, reaches a maximum just after the thermal activation of third-order intercone processes, then begins to decrease as the third-order conductivity increases sharply. Eventually the decrease stops as the first-order current drowns out the third order. Hence $b=0.5 \times 10^{8} \hbar$ and $b=10^{8} \hbar$ increase with temperature over the entire region, because the first-order current increases the most rapidly.

Although the lower $b$ curves increase with temperature they still provide the lowest critical fields, thanks to largermagnitude third-order current, as well as being the most robust to thermal fluctuations. These properties make them ideal from an applications standpoint.

Figure 12 shows that smaller chemical potentials produce smaller critical fields but increase with temperature at a larger rate. Over the temperature range surveyed, the critical field for $\mu_{0}=100 \mathrm{meV}$ only increases by $1 / 6$ of its original value; hence larger chemical potentials produce a worse but more stable nonlinear material.

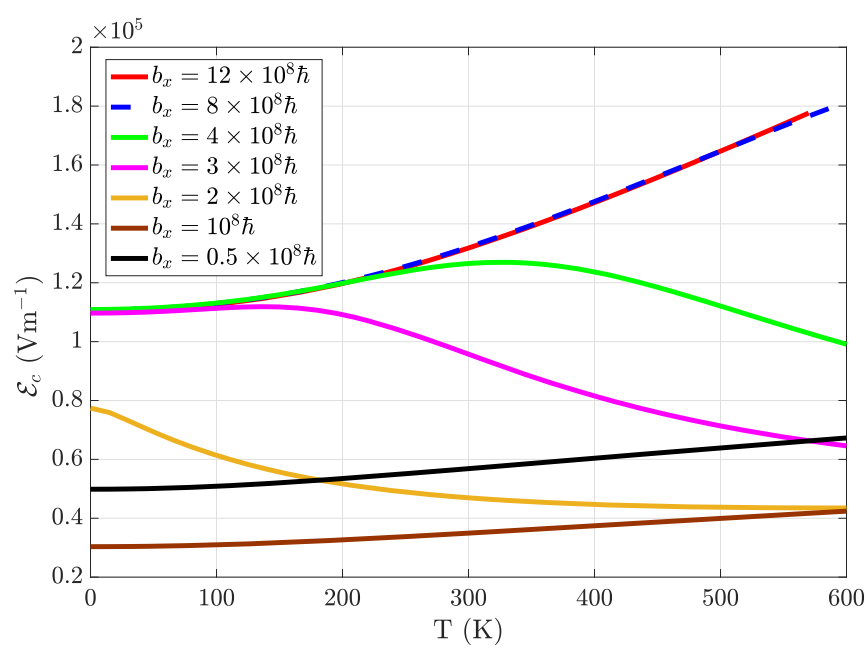

FIG. 11. Critical field with $\omega=1 \mathrm{THz}, \mu_{0}=80 \mathrm{meV}$, and $\Delta=$ $53 \mathrm{meV}$.

As expected from Eqs. (9) and (11), the temperature dependence of the critical field is not sensitive to $\Delta$ with all curves following the universal trend

$$
\frac{\mathcal{E}_{c}(T)}{\mathcal{E}_{c}(T=0)} \approx \sqrt{\frac{1+\alpha T^{2}}{\gamma+\gamma e^{-\delta T^{2}}}},
$$

where $\gamma \gg \delta>\alpha$ implies the critical field increases approximately exponentially with temperature for $b=b_{x}=8 \times$ $10^{8} \hbar$.

Finally we remark that all critical fields are of the order of $10^{4}-10^{5} \mathrm{~V} \mathrm{~m}^{-1}$ and hence experimentally accessible, classifyng any WSM with these parameters a practically viable nonlinear material as expected from previous results for similar materials (e.g., graphene) [34,36-38].

\section{E. Perpendicular fields}

In this section we consider the case of $\mathcal{E} \perp \mathbf{b}$ whereby the applied field drives the charge carriers in a direction

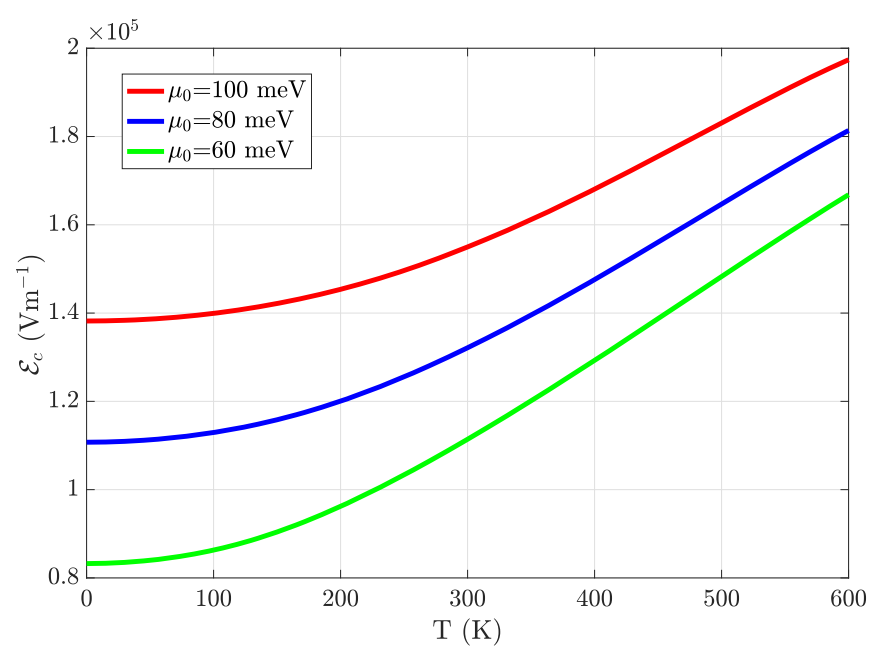

FIG. 12. Critical field with $\omega=1 \mathrm{THz}, b=b_{x}=8 \times 10^{8} \hbar$, and $\Delta=53 \mathrm{meV}$. 

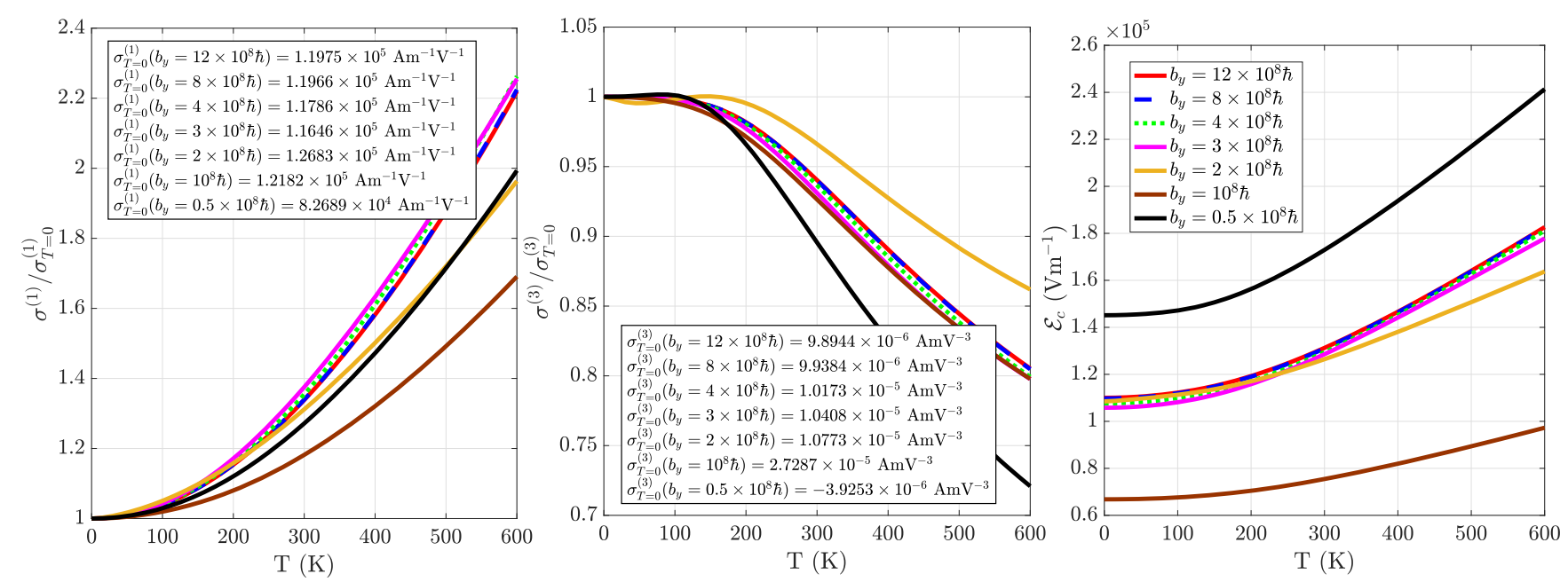

FIG. 13. Temperature dependence of the first-order conductivity (left), third-order conductivity (center), and critical field (right) for a WSM with cone separation perpendicular to the applied field and $\mu_{0}=80 \mathrm{meV}, \Delta=53 \mathrm{meV}$, and $\omega=1 \mathrm{THz}$ for the $\Delta=0 \mathrm{meV}$ values.

perpendicular to the cone separation. This ensemble is easily achieved by using $\mathbf{b}=\left(0, b_{y}, 0\right)$ and should highlight the anisotropy of the WSM dispersion.

The effects of changing $\mu_{0}$ mirror those seen in the parallel field case since the Fermi level variation is independent of the field direction relative to $\mathbf{b}$. Hence, we do not present different $\mu_{0}$ curves and instead restrict this analysis to the band gap and temperature dependence of WSMs with different $b$ values.

Figure 13 shows the temperature and band-gap dependence of the conductivity under a perpendicular field. In the first order, all $b$-value curves increase with temperature as expected. As $E_{s=1, r=-1}^{\mathrm{ex}}$ approaches zero, the rate of increase with temperature drops; this is expected since the charge carriers will, on average, exist in lower-energy states. Conversely, the $T=0 \mathrm{~K}$ conductivity magnitude increases as $b$ becomes smaller.

Meanwhile the magnitude of the third-order conductivity reaches a distinct peak when $v_{F} b=\Delta$ and $E_{s=1, r=-1}^{\mathrm{ex}}=0$. In fact it becomes negative for $b=0.5 \times 10^{8} \hbar$. Most notably, since there is minimal thermal activation of intercone transitions under a perpendicular field, the third-order conductivity will, in general, decrease with temperature as more charge carriers participate in single-photon processes. There are two small regions that are exceptions to this rule: $0-100 \mathrm{~K}$ for $b_{y}=$ $0.5 \times 10^{8} \hbar$ and $50-150 \mathrm{~K}$ for $b_{y}=2 \times 10^{8} \hbar$. We hypothesize that these are small regions of thermally activated intercone transitions since $v_{F} b$ is just larger $\left(b_{y}=2 \times 10^{8} \hbar\right)$ or smaller $\left(b_{y}=0.5 \times 10^{8} \hbar\right)$ than $\Delta$.

Although the magnitude of the third-order current is at its largest for $b_{y}=10^{8} \hbar$ whereby $E_{s=1, r=-1}^{\mathrm{ex}}=E_{F}$, the critical field also peaks for this value of $b$ owing to a large first-order current. Of the remaining $b$ values, despite $b_{y}=0.5 \times 10^{8} \hbar$ displaying the smallest critical field, we rank $b_{y}=2 \times 10^{8} \hbar$ as the best nonlinear material for higher temperatures and $b_{y}=12 \times 10^{8} \hbar$ as the best nonlinear material for lower temperatures in this field geometry. This is because $\sigma^{(3)}\left(b_{y}=\right.$ $\left.0.5 \times 10^{8} \hbar\right)$ is negative and hence detracts from the overall current response. Unfortunately, all curves increase with temperature and take values above $4 \times 10^{4} \mathrm{~V} \mathrm{~m}^{-1}$ which, with reference to Fig. 11, shows that the nonisotropic WSM is, in general, a better photo-mixer of fields directed parallel to $\mathbf{b}$, particularly at higher temperatures.

Figure 14 displays remarkably different properties from the parallel setup, revealing characteristic peaks in both the first and third order. First, there is a monotonic decrease in the $\Delta=0$ first-order conductivity values, as in the parallel field geometry. However, unlike the parallel geometry, when $v_{F} b \approx \Delta$ there is not just an inflection but an increase in the first-order conductivity as $E_{s=1, r=-1}^{\mathrm{ex}}$ decreases towards zero, just prior to which the conductivity maximum is attained and after which it decreases once again. The height of each maxima increases with $b$, but occurs at a larger $\Delta$.

The third-order curves display much sharper peaks exactly when $v_{F} b=\Delta$ when the lowest (rest) energy charge carriers possible participate in third-order processes. Contrary to the first order and the parallel field geometry, the $\Delta=0$ conductivities increase with $b$ due to $E_{s=1, r=-1}^{\mathrm{ex}}$ decreasing monotonically with $b$ for $\Delta=0$. Despite this, the heights of each peak still increase with $b$. After each curve has reached its peak the conductivity drops to a negative value then decreases in magnitude towards zero as the band gap increases.

The third-order conductivity peak for $v_{F} b=\Delta$ characterizes the critical field minimum. The immediate peak thereafter is a discontinuity as $\sigma^{(3)}$ crosses zero, after which the critical field increases at an exponential rate independent of $b$ as $\sigma^{(3)}$ approaches zero. The minima of each curve are equal as the material limit is reached, much the same as the coincident minima for $b_{x}=10^{8} \hbar$ and $b_{x}=0.5 \times 10^{8} \hbar$ in Fig. 10 .

For consistency we note that all dynamics for which $v_{F} b<$ $\Delta$ (fully gapped case) are identical in the perpendicular and parallel cases owing to the isotropic dispersion.

\section{F. Effect of finite relaxation}

Until now we have neglected the role that relaxation plays in the transport properties discussed as our emphasis is the band-induced photon mixing. In reality, there is a finite relaxation due to various disorders. In the present case 

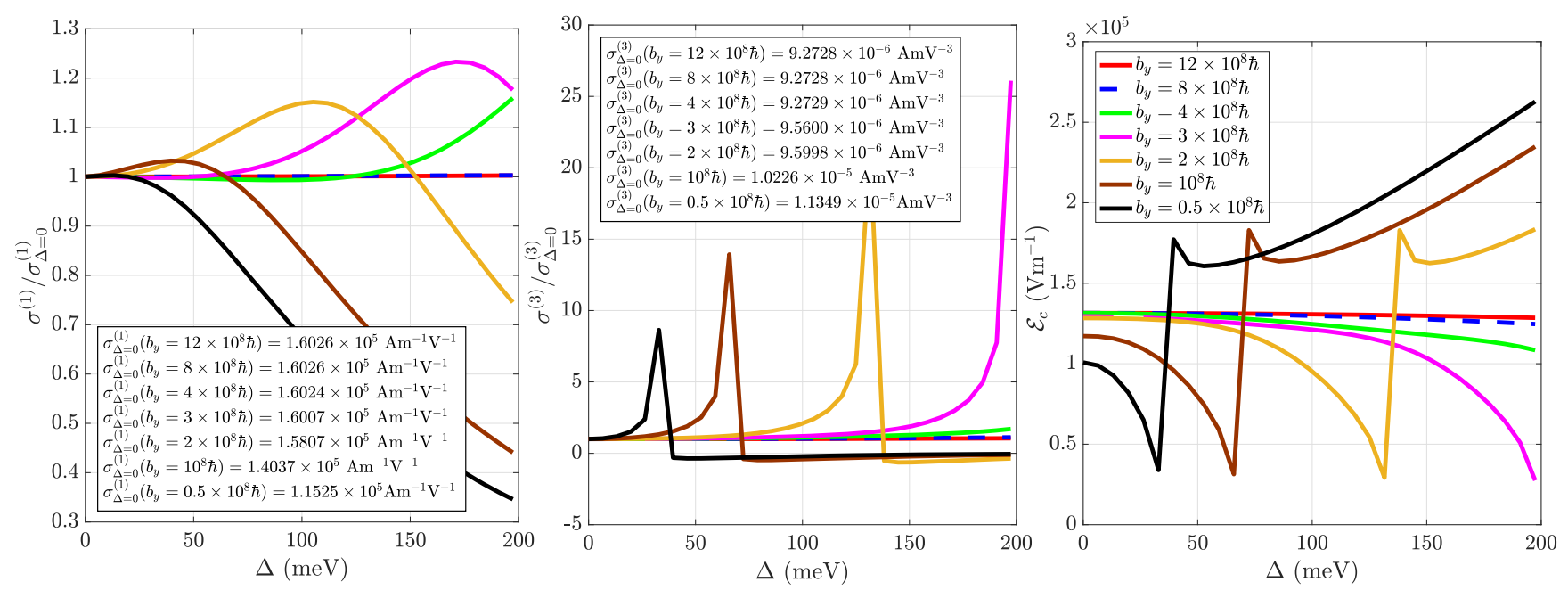

FIG. 14. Band-gap dependence of the first-order conductivity (left), third-order conductivity (center), and critical field (right) for a WSM with cone separation perpendicular to the applied field and $\mu_{0}=80 \mathrm{meV}, T=300 \mathrm{~K}$, and $\omega=1 \mathrm{THz}$ for the $\Delta=0 \mathrm{meV}$ values.

of a gapped system, the relaxation is finite since the gap itself is due to some kind of disorder. To have a qualitative discussion on the relaxation, we use the constant relaxation time approximation, $\omega \rightarrow \omega+i \gamma$, where $\gamma$ is the relaxation rate. Since the vector potential is $\propto 1 /(\omega+i \gamma)$ and it encodes all temporal information into the current response via $J_{x}^{(n)}=\sigma_{x}^{(n)} \mathcal{E}^{n} \propto A_{x}^{n}$, by normalizing all $\sigma_{x}^{(n)}$ curves to their $\Delta=0$ or $T=0$ values, introducing relaxation into the model via $\omega \rightarrow \omega+i \gamma$ will not change any earlier results. Instead, to see the effect relaxation plays in this model, we calculate

$$
\begin{aligned}
& \frac{J_{x}^{(1)}}{J_{x}^{(1), \operatorname{Rel}}}=\frac{\omega+i \gamma}{\omega}, \\
& \frac{J_{x}^{(3)}}{J_{x}^{(3), \operatorname{Rel}}}=\left[\left(1-\frac{3 \gamma^{2}}{\omega^{2}}\right)+i\left(\frac{3 \gamma^{2} \omega-\gamma^{3}}{\omega^{3}}\right)\right] \text {. }
\end{aligned}
$$

Overall Fig. 15 shows that, unless the relaxation parameter $\gamma$ is comparable to the applied field frequency $\omega$, relaxation plays a minimal role in this model since the real (red) and absolute value (black) curves are close to unity while the imaginary part (blue) is close to zero. In most Dirac materials the relaxation time is typically on the order of $10 \mathrm{ps}$. For an external field with frequency of $1-5 \mathrm{THz}, \omega \tau$ is between 10 and 50. Therefore, the high-order terms in $1 / \omega \tau$ are small quantities. We also note that our approximation neglects the energy-dependent relaxation. The frequency dependence is negligible since there is no resonant scattering around $1 \mathrm{THz}$. It has been shown that this relaxation approximation is valid for several different Dirac systems [39-42]. For strong scattering, an approach beyond the relaxation approximation will be required. For example, the self-consistent Born approximation will give a better description.

The imaginary parts of both the first- and third-order conductivity ratios increase with $\gamma$. Since the real part of the first-order ratio is unity, this produces an increase in the absolute value of the ratio. Conversely, in the third order, both the real part and the absolute value of the conductivity ratio decrease with $\gamma$, with the absolute value decreasing at a slightly slower rate owing to the growth in the imaginary component. Physically these results imply that a finite relaxation hinders the first-order conductivity slightly, while actually enhancing the third-order conductivity slightly.

\section{CONCLUSION}

We have applied a semiclassical formalism to calculate the temperature and band-gap dependence of the linear and nonlinear conductivities of a WSM. The sensitivity of these conductivities to applied field geometry and Weyl point separation have also been determined.

Our results indicate that the relative magnitudes of $v_{F} b$ and $\Delta$ characterize the $\Delta$ dependence while the relative magnitudes of $E_{F}$ and $E_{s=1, r=-1}^{\mathrm{ex}}$ characterize the temperature dependence. In general, when $v_{F} b \approx \Delta$ characteristic peaks or points of inflection are observed in the $\Delta$ dependence. The most notable peak is for the third-order conductivity when $\Delta$ just becomes larger than zero. This peak is topologically induced and produces a highly desirable small critical field. Similarly, characteristic minima are observed in the temperature dependence when $E_{F} \approx E_{s=1, r=-1}^{\mathrm{ex}}$.

For $\Delta>v_{F} b$ the third-order conductivity drops off more rapidly than the first order with $\Delta$ due to the heightened difficulty of multiple-photon processes. Conversely at higher temperatures whereby $E_{F}>E_{s=1, r=-1}^{\mathrm{ex}}$ the third-order conductivity is thermally enhanced up to five times its initial $T=0 \mathrm{~K}$ value at $600 \mathrm{~K}$ for $b=b_{x}=2 \times 10^{8} \hbar$ if the applied field drives the charge carrier parallel to $\mathbf{b}$. However, if the field instead drives the charge carriers in a direction perpendicular to $\mathbf{b}$ there is a thermal reduction of the third-order conductivity.

Although the overall magnitudes of the conductivities follow expected patterns, we reveal that the $\Delta$ dependence of both the first- and third-order dependence are independent of chemical potential and temperature. There is a further independence on $\Delta$ observed in the temperature dependence for both the first and third orders. 

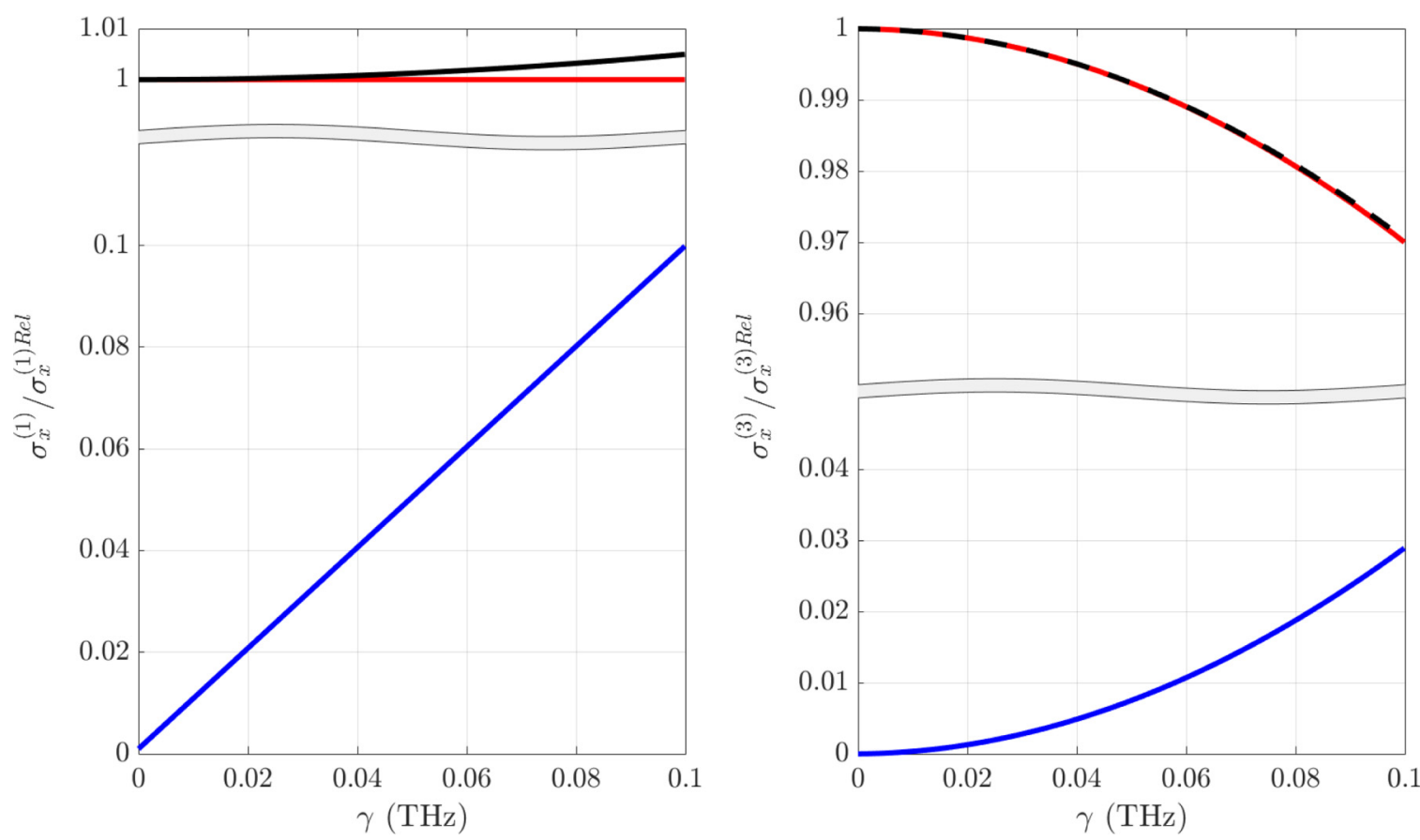

FIG. 15. The effect of relaxation in the model. The ratios of the first-order conductivity (left) and third-order conductivity (right) are plotted with and without relaxation present. All red curves represent the real parts of the ratio, the blue curves represent the imaginary parts, and the black curves the absolute value. All plots have $\omega=1 \mathrm{THz}$.

One of the most striking findings of this study is that there is no nonlinear response if $v_{F} b=\Delta=0$ due to the spherical symmetry of the higher-order velocity operators. This is in stark contrast to the two-dimensional case (graphene) where the third-order response is appreciable for low applied fields.

In Sec. III F we show that including a finite relaxation time in this model changes the conductivities very minimally so long as the relaxation rate is much less than the applied field frequency. The minimal trend observed, however, is that a finite relaxation time will effectively increase the nonlinearity of a WSM by around $0.5 \%$ for $\gamma=0.1 \omega$.

In practice, the $\Delta$ dependence could prove useful in providing a purely conductivity-based method of determining $b$ experimentally, alleviating the need for more difficult field geometries or measuring Fermi arcs. Finally, the low critical field values of $10^{4}-10^{5} \mathrm{~V} \mathrm{~m}^{-1}$ coupled with the ability to strongly tune the nonlinear response by altering temperature or $\Delta$ (by doping) strongly bolster the case for using WSMs as photo-mixers in the terahertz range.
We note that our semiclassical approach captures the photo-mixing features due to the band structure of the system. While the average velocity for a given state in our formalism is equivalent to that calculated by using quantum mechanical wavefunctions, the formalism does not have explicit dependence on the quantum mechanical wavefunction. A quantum formalism based on Floquet states can also be used to treat the problem of electrons coupled to a photon field. Using Floquet states or a Magnus expansion one can construct the full density matrix which includes all orders of electron-photon coupling [43-45].

\section{ACKNOWLEDGMENTS}

This work is supported by the National Key Research and Development Program of China (Grants No. 2018YFF01013001 and No. 2017YFA0701000), the Natural Science Foundation of China (Grants No. 61701084 and No. 61505022), and by the Australian Research Council (Grant No. DP160101474).
[1] S. Y. Xu, I. Belopolski, N. Alidoust, M. Neupane, G. Bian, C. Zhang, R. Sankar, G. Chang, Z. Yuan, C. C. Lee, and S. M. Huang, Science 349, 613 (2015).

[2] B. Q. Lv, N. Xu, H. M. Weng, J. Z. Ma, P. Richard, X. C. Huang, L. X. Zhao, G. F. Chen, C. E. Matt, F. Bisti, and V. N. Strocov, Nat. Phys. 11, 724 (2015).

[3] L. Lu, Z. Wang, D. Ye, L. Ran, L. Fu, J. D. Joannopoulos, and M. Soljačić, Science 349, 622 (2015)

[4] S. Y. Xu, N. Alidoust, I. Belopolski, Z. Yuan, G. Bian, T. R. Chang, H. Zheng, V. N. Strocov, D. S. Sanchez, G. Chang, and C. Zhang, Nat. Phys. 11, 748 (2015).
[5] T. Ouyang, H. Xiao, C. Tang, M. Hu, and J. Zhong, Phys. Chem. Chem. Phys. 18, 16709 (2016).

[6] T. Meng and L. Balents, Phys. Rev. B 86, 054504 (2012).

[7] P. Hosur, S. A. Parameswaran, and A. Vishwanath, Phys. Rev. Lett. 108, 046602 (2012).

[8] P. Goswami and S. Tewari, Phys. Rev. B 88, 245107 (2013).

[9] M. M. Vazifeh and M. Franz, Phys. Rev. Lett. 111, 027201 (2013).

[10] N. P. Armitage, E. J. Mele, and A. Vishwanath, Rev. Mod. Phys. 90, 015001 (2018).

[11] P. E. Ashby and J. P. Carbotte, Phys. Rev. B 87, 245131 (2013). 
[12] J.-N. Fuchs, arXiv:1306.0380.

[13] B. Q. Lv, H. M. Weng, B. B. Fu, X. P. Wang, H. Miao, J. Ma, P. Richard, X. C. Huang, L. X. Zhao, G. F. Chen, and Z. Fang, Phys. Rev. X 5, 031013 (2015).

[14] A. C. Neto, F. Guinea, N. M. Peres, K. S. Novoselov, and A. K. Geim, Rev. Mod. Phys. 81, 109 (2009).

[15] N. M. R. Peres, Rev. Mod. Phys. 82, 2673 (2010).

[16] S. Das Sarma, S. Adam, E. H. Hwang, and E. Rossi, Rev. Mod. Phys. 83, 407 (2011).

[17] P. S. Baireuther, Doctoral dissertation, Lieden University, 2017.

[18] J. S. Bell and R. Jackiw, Nuovo Cimento A 60, 47 (1969).

[19] S. L. Adler, Phys. Rev. 177, 2426 (1969).

[20] D. T. Son and B. Z. Spivak, Phys. Rev. B 88, 104412 (2013).

[21] K. Landsteiner, Phys. Rev. B 89, 075124 (2014).

[22] C. Shekhar, A. K. Nayak, Y. Sun, M. Schmidt, M. Nicklas, I. Leermakers, U. Zeitler, Y. Skourski, J. Wosnitza, Z. Liu, and Y. Chen, Nat. Phys. 11, 645 (2015).

[23] T. Ando and T. Nakanishi, J. Phys. Soc. Jpn. 67, 1704 (1998).

[24] M. I. Katsnelson and M. I. Katsnelson, Graphene: Carbon in Two Dimensions (Cambridge University Press, Cambridge, UK, 2012).

[25] A. V. Balatsky, I. Vekhter, and J. X. Zhu, Rev. Mod. Phys. 78, 373 (2006).

[26] F. Evers and A. D. Mirlin, Rev. Mod. Phys. 80, 1355 (2008).

[27] G. Xu, H. Weng, Z. Wang, X. Dai, and Z. Fang, Phys. Rev. Lett. 107, 186806 (2011).

[28] K. Y. Yang, Y. M. Lu, and Y. Ran, Phys. Rev. B 84, 075129 (2011).

[29] D. E. Parker, T. Morimoto, J. Orenstein, and J. E. Moore, Phys. Rev. B 99, 045121 (2019).
[30] L. Wu, S. Patankar, T. Morimoto, N. L. Nair, E. Thewalt, A. Little, J. G. Analytis, J. E. Moore, and J. Orenstein, Nat. Phys. 13, 350 (2017).

[31] S. Patankar, L. Wu, B. Lu, M. Rai, J. D. Tran, T. Morimoto, D. E. Parker, A. G. Grushin, N. L. Nair, J. G. Analytis, and J. E. Moore, Phys. Rev. B 98, 165113 (2018).

[32] S. Ahn, E. J. Mele, and H. Min, Phys. Rev. B 95, 161112(R) (2017).

[33] C. Hwang, D. A. Siegel, S. K. Mo, W. Regan, A. Ismach, Y. Zhang, A. Zettl, and A. Lanzara, Sci. Rep. 2, 590 (2012).

[34] S. Shareef, Y. S. Ang, and C. Zhang, J. Opt. Soc. Am. B 29, 274 (2012).

[35] L. Tapaszto, G. Dobrik, P. Nemes-Incze, G. Vertesy, P. Lambin, and L. P. Biro, Phys. Rev. B 78, 233407 (2008).

[36] A. R. Wright, X. G. Xu, J. C. Cao, and C. Zhang, Appl. Phys. Lett. 95, 072101 (2009).

[37] Y. S. Ang and C. Zhang, J. Phys. D: Appl. Phys. 45, 395303 (2012).

[38] S. A. Mikhailov, Europhys. Lett. 79, 27002 (2007).

[39] N. M. R. Peres, Yu. V. Bludov, J. E. Santos, A-P. Jauho, and M. I. Vasilevskiy, Phys. Rev. B 90, 125425 (2014).

[40] S. Nandy, G. Sharma, A. Taraphder, and S. Tewari, Phys. Rev. Lett. 119, 176804 (2017).

[41] A. Cortijo, Phys. Rev. B 94, 235123 (2016).

[42] S. A. Mikhailov and K. Ziegler, Phys. Rev. Lett. 99, 016803 (2007).

[43] S. Blanes, F. Casas, J. A. Oteo, and J. Ros, Phys. Rep. 470, 151 (2009).

[44] S. Restrepo, J. Cerrillo, V. M. Bastidas, D. G. Angelakis, and T. Brandes, Phys. Rev. Lett. 117, 250401 (2016).

[45] T. Kuwahara, T. Mori, and K. Saito, Ann. Phys. (NY) 367, 96 (2016). 